\title{
Inverse Problem Solution for Quantitative Investigations of Nanocrystals Formation and Growth
}

Anton V. Ievlev ${ }^{1,2}$, Stephen Jesse ${ }^{1,2}$, Vighter Iberi ${ }^{1,2}$, Thomas J. Cochell ${ }^{3}$, Vladimir A. Protopopescu ${ }^{1,4}$, Raymond R. Unocic $^{1,2}$, Olga Ovchinnikova ${ }^{1,2}$, Sergei V. Kalinin ${ }^{1,2}$

${ }^{1}$ Center for Nanophase Materials Sciences, Oak Ridge National Laboratory, Oak Ridge, TN, 37831

${ }^{2}$ Institute for Functional Imaging of Materials, Oak Ridge National Laboratory, Oak Ridge, TN 37831

${ }^{3}$ Chemical and Materials Engineering, University of Kentucky, Lexington, KY 40506

${ }^{4}$ Computational Sciences\&Engineering Division, Oak Ridge National Laboratory, Oak Ridge, TN 37831

Nanoparticles and nanocrystals remain one of the central objects of study in material science, physics and chemistry. Their unique intrinsic properties enable a great number of practical applications, including semiconductor and magnetic devices, materials for energy storage, generation, catalysis and biomedicine, but also remain of great interest for fundamental science [1-3]. However processes, defining nanostructures nucleation and growth still require investigations. Recently developed experimental techniques (e.g. liquid cell STEM) provide tools for direct in-situ visualization of these processes with nanometer and even sub-nanometer spatial resolution [4]. However, systematic analysis of the acquired multidimensional data ( 2 spatial and 1 time dimensions) represents a nontrivial, complex problem. Most studies performed to date are limited by qualitative discussions, visualization of the particles and macroscopic averaging of the system parameters, while local features of the growth, collective dynamics and proximity effects remain poorly investigated. However, this data can provide important information about physical and chemical phenomena underpinning system behavior, which can be used for optimizing the condition of the synthesis process.

Here, we developed a framework [5] for the systematic analysis and interpretation of time-resolved observations of platinum nanocrystal nucleation and growth induced by electron and He-ion beams (Fig. 1a). We introduced three levels of data analysis and interpretation, including: average statistical; network; and local levels. In particular, this allowed detection of the local particle dynamics features (Fig. 2c) and interaction between individual particles (Fig. 2b). We further developed a local approach for inverse problem solution to correlate experimentally observed behavior with fundamental physics and chemistry. The approach is based on numerical simulations with experimental system geometry and comparison of simulated fields (Pt concentration, electric field, etc.) with experimentally observed dynamics of the growth. Application of this approach to analysis of the platinum nanocrystal formation showed principal differences in origin of the system behavior between electron and He-ion induced growth [6].

\section{References:}

[1] Peng X., et.al. Nature, 404 (2000), p.59

[2] Gupta A.K, Gupta M. Biomaterials, 26, (2005), p.3995.

[3] Jacob M., Levanon H, Kamat P.V., Nano letters, 3, (2003), p. 353.

[4] HG Liao, K Niu, and H Zheng. Chemical Communications 49 (2013) p. 11720.

[5] Ievlev, A.V., et.al, ACS Nano, 9(12), (2015), p. 11784

[6] Research supported by Oak Ridge National Laboratory's Center for Nanophase Materials Sciences (CNMS), which is a U.S. Department of Energy, Office of Science User Facility. 

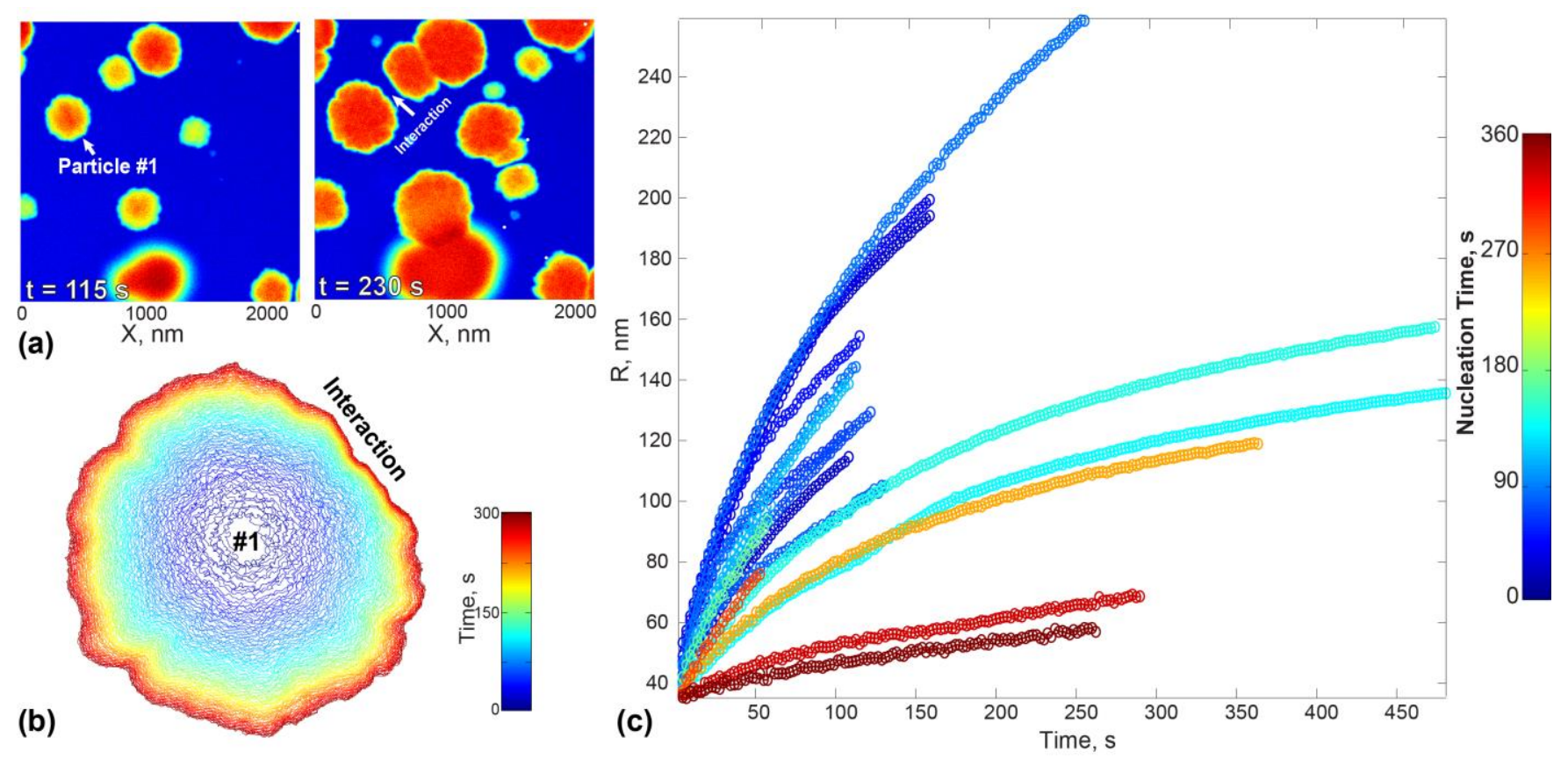

(b)
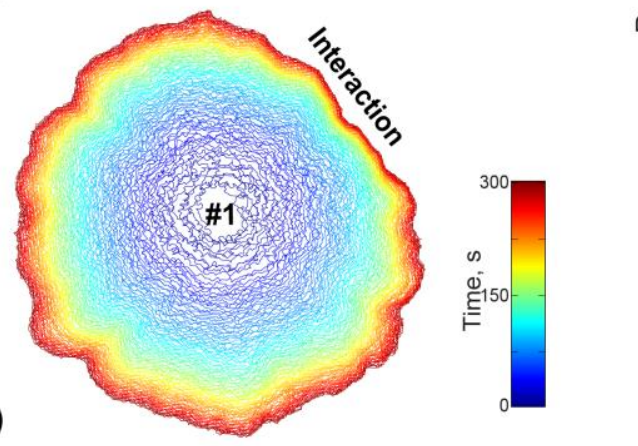

Figure 1. Analysis of the Pt nanoparticles nucleation and growth induced by electron beam of STEM. (a) Still frames of the particle growth; (b) Time evolution of the single particle fluid-solid interface boundary; (c) Time plot of the particles radius as a function of nucleation time (curve color). 